\title{
Impact of Hepatic Steatosis on the Antiviral Effects of PEG-IFN $\alpha$-2a in Patients with Chronic Hepatitis B and the Associated Mechanism
}

\author{
Huiqing Liang, ${ }^{1,2}$ Yaoyu Liu, ${ }^{3}$ Xiaoqian Jiang, ${ }^{3}$ Xiaoting Zheng, ${ }^{3}$ Jinmo Tang, ${ }^{1}$ Jiaen Yang, \\ Hongli Zhuang, ${ }^{4}$ Penghua Lai, ${ }^{2}$ Li Peng, ${ }^{2}$ Zhenying Guo, ${ }^{2}$ Shanshan Cai, ${ }^{3}$ Dan Luo, ${ }^{3}$ \\ Lingxia Xu, ${ }^{2}$ Qianguo Mao ${ }^{1}{ }^{1}$, and Shaodong Chen $\mathbb{D D}^{2}$ \\ ${ }^{1}$ Hepatology Unit, Xiamen Hospital of Traditional Chinese Medicine, Xiamen, Fujian, China \\ ${ }^{2}$ Department of Traditional Chinese Medicine, Medical College of Xiamen University, Xiamen, Fujian, China \\ ${ }^{3}$ Fujian University of Traditional Chinese Medicine, Fujian, China \\ ${ }^{4}$ Department of Traditional Chinese Medicine, No.1 Hospital Affiliated to Xiamen University, Xiamen, Fujian, China \\ Correspondence should be addressed to Qianguo Mao; zhuzhuq3@tom.com and Shaodong Chen; adong@xmu.edu.cn
}

Received 11 July 2019; Revised 18 March 2020; Accepted 30 March 2020; Published 26 June 2020

Academic Editor: Amosy M'Koma

Copyright (C) 2020 Huiqing Liang et al. This is an open access article distributed under the Creative Commons Attribution License, which permits unrestricted use, distribution, and reproduction in any medium, provided the original work is properly cited.

\begin{abstract}
Objective. To investigate the risk factors for hepatic steatosis in chronic hepatitis B (CHB), to determine its correlation with liver necroinflammation and fibrosis and response to peginterferon alpha-2a (PEG-IFN $\alpha-2 \mathrm{a}$ ) antiviral therapy, and to explore the mechanisms underlying the poor antiviral effect of PEG-IFN $\alpha-2 \mathrm{a}$ in CHB patients with hepatic steatosis. Methods. We analysed the impact of hepatic steatosis on the antiviral effect of PEG-IFN $\alpha-2 \mathrm{a}$ on CHB patients in a cohort of 226 patients who underwent pretherapeutic liver biopsy. To assess the complete response (CR), virological response (VR), and biochemical response (BR), the 226 patients were treated with PEG-IFN $\alpha$-2a for 48 weeks and were followed-up for 24 weeks. The expressions of hepatitis B surface antigen $(\mathrm{HBsAg})$ and hepatitis B core antigen $(\mathrm{HBcAg})$ in the liver tissue were detected in all patients to explore the possible mechanism of hepatic steatosis with regard to antiviral effects. Results. The patients were divided into four groups based on the severity of hepatic steatosis: 119 with no steatosis, 76 with mild steatosis, 22 with moderate steatosis, and 9 with severe steatosis. In the hepatic steatosis groups, the proportions of male patients, patients aged $>40$ years, patients with hyperuricaemia, patients with a $\mathrm{BMI}>23 \mathrm{~kg} / \mathrm{m}^{2}$, and total cholesterol (TC), triglyceride (TG), glucose (GLU), and uric acid (UA) levels were significantly higher than those in the group without steatosis, whereas the alanine aminotransferase (ALT) and aspartate transaminase (AST) levels were significantly lower than those in the group without steatosis. The multivariate analysis results indicated that a BMI $>23 \mathrm{~kg} / \mathrm{m}^{2}$ was independently associated with CHB patients with hepatic steatosis; the levels of baseline AST and UA were independently associated with CHB patients with significant hepatic steatosis, and the baseline AST level was independently associated with significant liver fibrosis. After 48 weeks of treatment and 24 weeks of follow-up, the rates of CR, VR, and BR had gradually decreased, whereas the severity of hepatic steatosis had increased. Conclusion. Hepatic steatosis can reduce the efficacy of PEG-IFN $\alpha-2 \mathrm{a}$ in the treatment of CHB patients, and its mechanism may be related to the different $\mathrm{HBcAg}$ expression patterns in liver tissue.
\end{abstract}

\section{Introduction}

Chronic hepatitis $\mathrm{B}(\mathrm{CHB})$ and nonalcoholic fatty liver disease (NAFLD) are two major chronic liver diseases in China. As lifestyle and dietary structure have changed, the number of $\mathrm{CHB}$ patients with hepatic steatosis has increased. The incidence varies widely around the world, fluctuating between 27 and $51 \%[1,2]$, with a median prevalence of 28\%. A report of 1915 liver biopsy cases in China revealed that the incidence of hepatic steatosis in $\mathrm{CHB}$ patients was $14 \%$ [3]. Whether CHB with hepatic steatosis has an aggravating effect on the progression of liver disease is unknown. 
A study in which liver biopsies were performed in $64 \mathrm{CHB}$ patients with hepatic steatosis by foreign scholars revealed that $86 \%$ of patients had varying degrees of fibrosis [4]. Among them, 39\% had progressive hepatic fibrosis, but no control group was established. In a study of $164 \mathrm{CHB}$ patients, Altlparmak et al. [5] compared patients with and without hepatic steatosis and suggested that there was no significant difference between the two groups in inflammation and fibrosis scores. Another study by Chen and Shi [6] reported that the degree of hepatic steatosis in $\mathrm{CHB}$ patients was negatively correlated with liver inflammation and fibrosis. Data are scarce regarding the impact of hepatic steatosis on antiviral treatments, particularly in terms of peginterferon alpha-2a (PEG-IFN $\alpha$-2a) treatment for CHB. A few earlier studies had revealed no significant association of hepatic steatosis with the antiviral effect in $\mathrm{CHB}$ patients [7, 8]. However, some new studies have shown that hepatic steatosis may reduce the antiviral efficacy of treatments for $\mathrm{CHB}$ patients [9-11]. Future studies examining the potential relationship between hepatic steatosis and the antiviral effect in CHB patients are necessary. This prospective cohort study assessed four different degrees of hepatic steatosis in $\mathrm{CHB}$ patients to investigate the effects of hepatic steatosis on $\mathrm{CHB}$ pathology and whether hepatic steatosis affects the efficacy of PEGIFN $\alpha-2$ a. Therefore, this study was conducted to compare the expression patterns of hepatitis B surface antigen (HBsAg) and hepatitis B core antigen ( $\mathrm{HBcAg}$ ) in hepatocytes in different groups and to explore the possible mechanism by which hepatic steatosis affects the efficacy of PEG-IFN $\alpha$-2a.

\section{Materials and Methods}

2.1. Patients. This prospective cohort study included 245 naive $\mathrm{CHB}$ patients who underwent percutaneous liver biopsy from May 2010 to 2016 at the Xiamen Hospital of Traditional Chinese Medicine. All patients underwent percutaneous liver biopsy, and their hepatic tissues were confirmed to exhibit or not exhibit steatosis by liver histological pathological examination. Patients were treated with PEG-IFN $\alpha$ 2a for 48 weeks and followed up for 24 weeks. Six patients were excluded due to abnormal thyroid function, eight patients were excluded due to an absolute neutrophil count in the peripheral blood $\leq 0.5 \times 10^{9} / \mathrm{L}$, and 5 patients were lost to follow-up due to emigration. The remaining 226 patients formed the study cohort. The patients were divided into four groups based on the severity of hepatic steatosis: 119 had no steatosis (group S0), 76 had mild steatosis (group S1), 22 had moderate steatosis (group S2), and 9 had severe steatosis (group S3). All patients were defined as HBsAg- and $\mathrm{HBcAg}$-positive for at least 6 months, with baseline $\mathrm{HBV}$ DNA levels greater than 20,000 IU/mL, ALT levels elevated between 2 and 10 times the upper limit of normal (ULN), and hepatic histopathology results demonstrating steatosis. Patients with a history of alcohol intake $>140 \mathrm{~g} /$ week, concomitant aetiologies including chronic hepatitis $\mathrm{C}$ or $\mathrm{D}$ coinfection or superinfection, autoimmune hepatitis, druginduced liver injury, HIV coinfection, or evidence of immune suppression were excluded. The study protocol was approved by the Ethics Committee of the Xiamen Hospital of Tradi- tional Chinese Medicine. All patients provided informed consent. Baseline pretreatment evaluations included collecting data on age, sex, course of disease, height, and weight, liver inflammation activity, fibrosis stage [12]; measurement of the HBV DNA levels; detection of HBV genotype; and quantification of the $\mathrm{HBV}$ e antigen (HBeAg), $\mathrm{HBsAg}$, ALT, AST, CHO, TG, GLU, and UA levels. All data were assessed in the central laboratory of the Xiamen Hospital of Traditional Chinese Medicine. The HBV DNA load was assessed by fluorescence quantitative PCR with a low detection limit of $500 \mathrm{IU} / \mathrm{mL}$ using the COBAS TaqMan HBV Test (Roche's Lightcycler fluorescence quantitative PCR system, Shanghai, China). Quantification of the HBsAg, HBsAb, $\mathrm{HBeAg}$, and $\mathrm{HBeAb}$ levels was performed by an electrochemiluminescence immunoassay using a Roche Elecsys 2010 system (German Roche Diagnostics Ltd, Germany). HBV genotypes were assessed by the PCR reverse dot blot hybridization method (Shenzhen Yaneng Biotechnology Co., Ltd).

2.2. Liver Biopsy and Histology. All patients provided informed consent for the liver biopsy procedure. The liver biopsy was performed using 16G biopsy needles guided by ultrasonography. A qualified biopsy specimen was either a minimum of $1.5 \mathrm{~cm}$ long or displayed for 6 or more portal tracts. The specimens were fixed, paraffin-embedded, and stained with haematoxylin and eosin (HE staining). Scheuer's scoring system was used to semiquantify the histological necroinflammation from G0 to G4 and the fibrosis stages from F0 to F4 by two pathologists independently who were blinded to the patients' biochemical and virologic results. Fibrosis was evaluated in all specimens by subjecting them to Masson Trichrome staining. Biopsied tissue sections were cut into $4 \mathrm{~mm}$ samples from prepared representative tissue blocks (lumps) and then placed in a paraffin oven to remove most of the paraffin. To complete deparaffinization, the specimens were then passed through xylene and a series of alcohol dilutions for 5 minutes and underwent microwave treatment using antigen retrieval for 10 minutes. After soaking in the methanol solution with $3 \% \mathrm{H}_{2} \mathrm{O}_{2}$ for 5 minutes to block endogenous peroxidase, the peroxidase-conjugated Envision Kit (Envision-PO, Envision System; DAKO, Carpinteria, CA, USA) for rabbit primary antibodies was applied to the specimens for immunohistochemical staining of $\mathrm{HBcAg}$ in the hepatocytes.

Hepatic steatosis was categorized as follows: no steatosis (steatosis affected less than 5\% of hepatocytes, S0), mild steatosis (steatosis affected $5-33 \%$ of hepatocytes, S1), moderate steatosis (steatosis affected $34-66 \%$ of hepatocytes, S2), and severe steatosis (steatosis affected more than $66 \%$ of hepatocytes, S3) (Figure 1) [13].

$\mathrm{HBcAg}$ expression patterns were classified as follows: no $\mathrm{HBcAg}$ expression (HBcAg-negative), cytoplasmic expression (cHBcAg), cytoplasmic dominant expression (cdHBcAg), and cytoplasmic and nuclear mean expression (mHBcAg).

The HBcAg-negative type showed no $\mathrm{HBcAg}$ expression in the nucleus or cytoplasm of the hepatocytes, the $\mathrm{cHBcAg}$ type showed HBcAg expression only in the cytoplasm but not in the nucleus of the hepatocytes, the cdHBcAg type showed more than $2 / 3$ of $\mathrm{HBcAg}$ expression in the cytoplasm, and the mHBcAg type showed 50\% HBcAg expression in the 


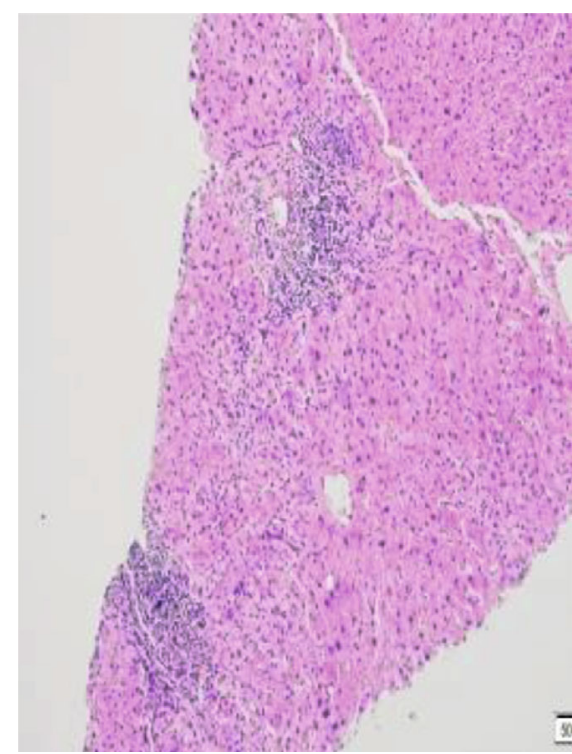

(a)

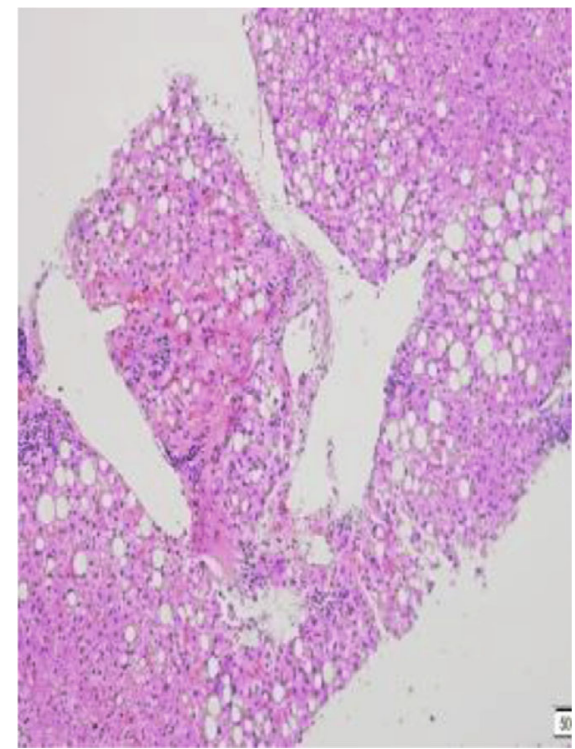

(c)

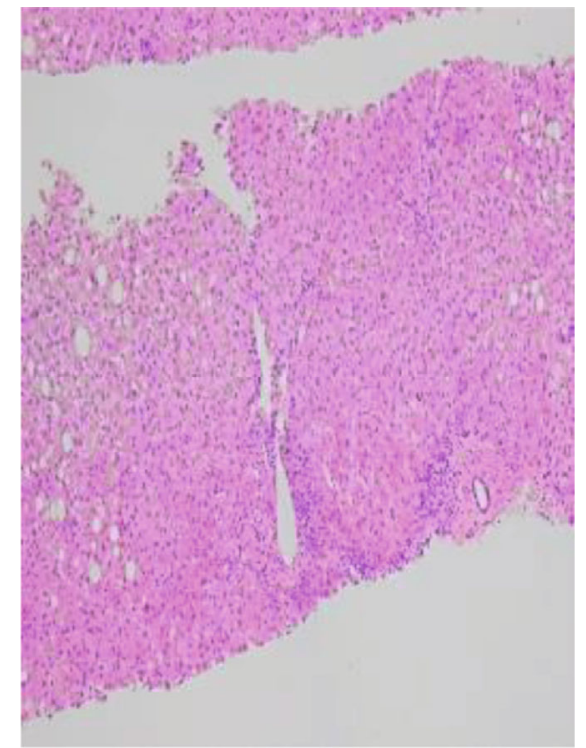

(b)

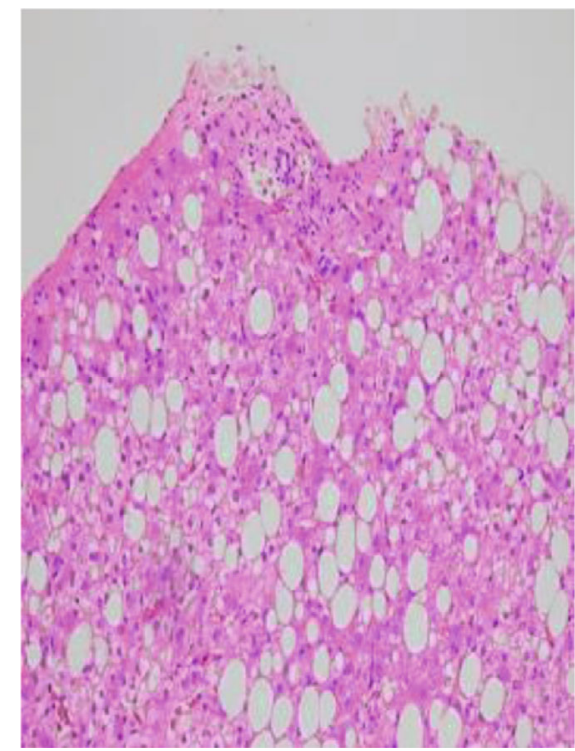

(d)

Figure 1: The distribution of hepatic steatosis was categorized into four types in this study. (a) In the S0 group, less than $5 \%$ of hepatocytes were affected by steatosis (HE staining, $\times 200$ ). (b) In the S1 group, 5-33\% of hepatocytes were affected by steatosis (HE staining, $\times 200)$. (c) In the S2 group, 34-66\% of hepatocytes were affected by steatosis (HE staining, $\times 200$ ). (d) In the S3 group, more than $66 \%$ of hepatocytes were affected by steatosis (HE staining, $\times 200$ ).

nucleus and $50 \%$ in the cytoplasm of the hepatocytes (Figure 2) [14].

$\mathrm{HBcAg}$ expression intensity was classified into four types in this study: 0 points, less than $5 \%$ hepatitis B core antigen expression; 1 point, $5 \%-33 \%$ hepatitis B core antigen expression; 2 points, $34 \%-66 \%$ hepatitis B core antigen expression; and 3 points, over $66 \%$ hepatitis B core antigen expression (Figure 3) [15].

The HBsAg expression intensity was classified into three types in this study: 1 point, $5 \%-33 \%$ hepatitis B surface antigen expression; 2 points, $34 \%-66 \%$ hepatitis B surface antigen expression; and 3 points, over $66 \%$ hepatitis B surface antigen expression (Figure 4) [15].
2.3. Antiviral Treatments. All patients were treated with $180 \mu \mathrm{g}$ iH of PEG-IFN $\alpha$-2a (Pegasys, Roche) once weekly for 48 weeks, and the dosage was adjusted according to any adverse effects identified by their physicians. After initiating the PEG-IFN $\alpha-2 \mathrm{a}$ treatment, the patients were followed-up at the outpatient clinics every 4 weeks. In addition to conventional biochemistry tests, the quantification of serum $\mathrm{HBsAg}$, anti-HBs, $\mathrm{HBeAg}$, anti-HBe, and HBV DNA was performed at baseline and at week 12 or 24,48 , and 72 . Complete response (CR) was defined as a serum HBV DNA level less than $500 \mathrm{IU} / \mathrm{mL}$, normal ALT levels $(<40 \mathrm{IU} / \mathrm{L})$, and $\mathrm{HBeAg}$ seroconversion (HBeAg loss and anti-HBe-positive) at 48 weeks posttreatment and 24 weeks follow-up. Virological 


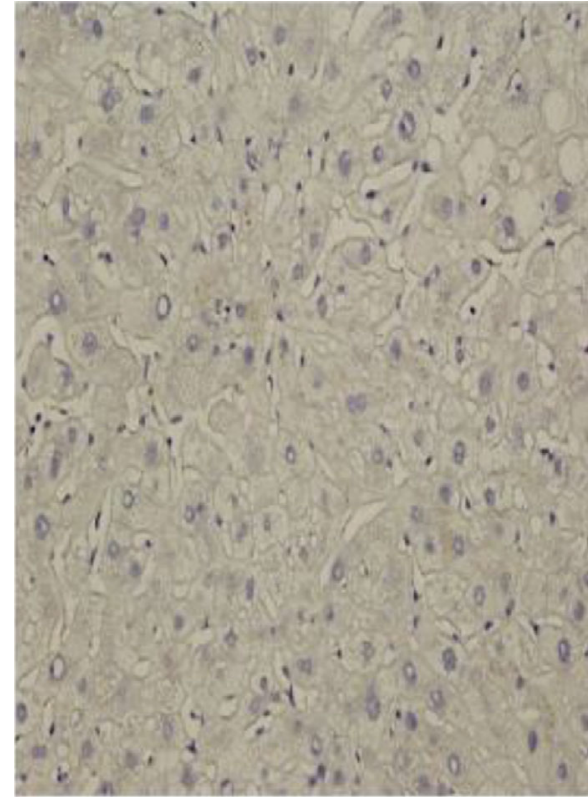

(a)

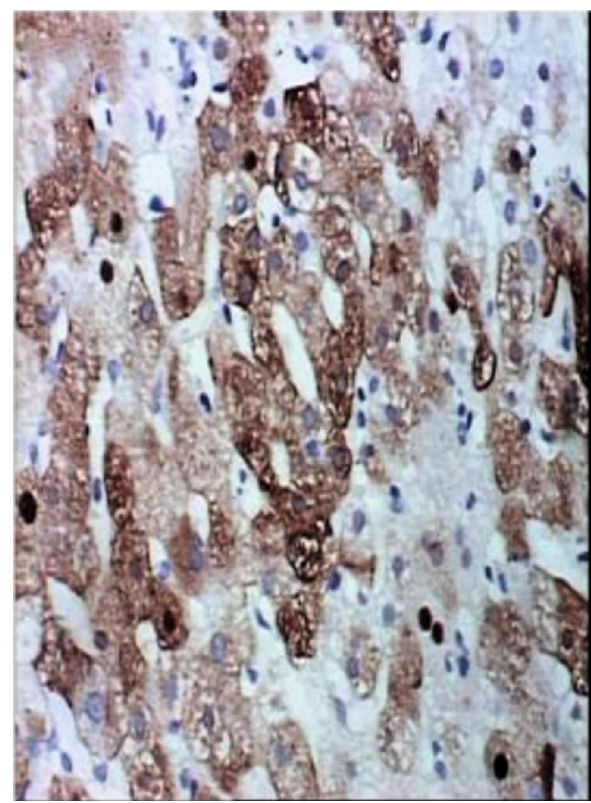

(c)

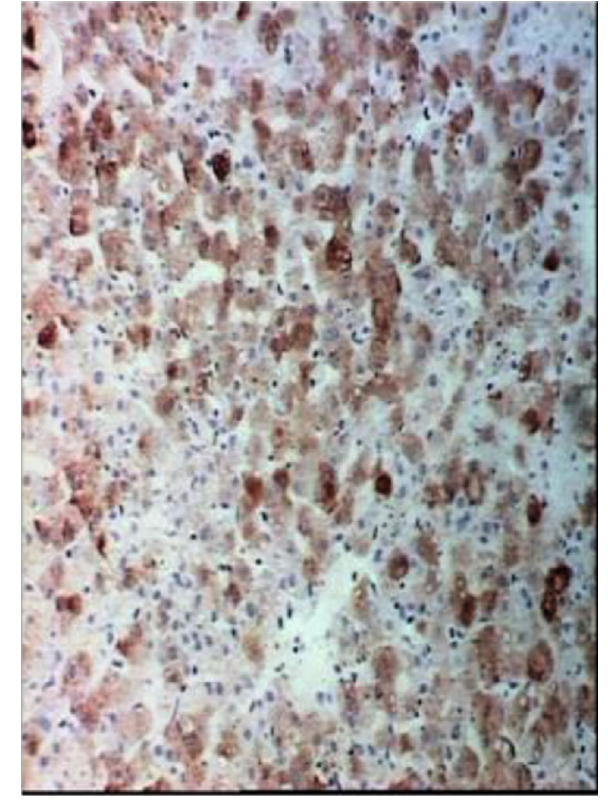

(b)

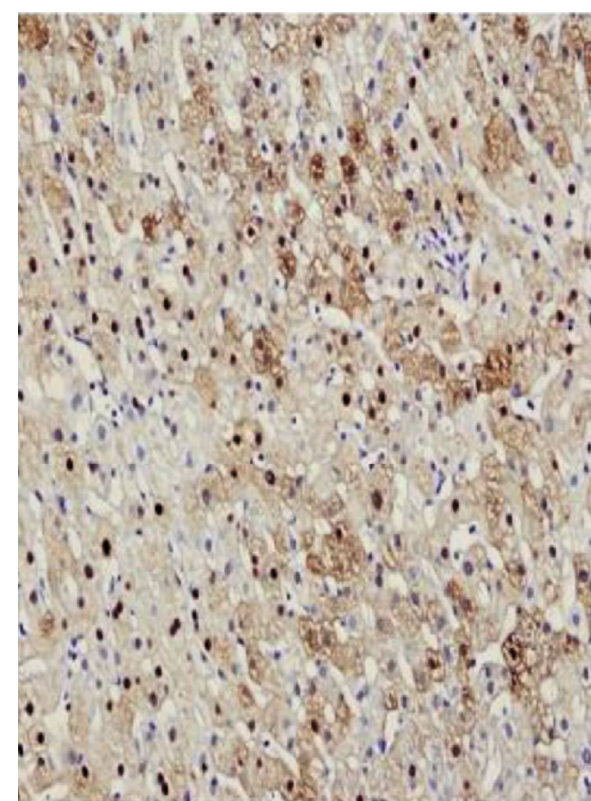

(d)

FIgURE 2: The distribution of $\mathrm{HBcAg}$ in the hepatocytes of CHB patients was classified into four types. (a) The HBcAg-negative type showed no $\mathrm{HBcAg}$ expression in either the nucleus or cytoplasm of the hepatocytes (immunohistochemical stain for $\mathrm{HBcAg}, \times 200)$. (b) The cHBcAg type showed HBcAg expression only in the cytoplasm but not in the nucleus of hepatocytes (immunohistochemical stain for $\mathrm{HBcAg}, \times 200$ ). (c) The cdHBcAg type showed more than $2 / 3 \mathrm{HBcAg}$ expression in the cytoplasm of hepatocytes (immunohistochemical stain for $\mathrm{HBcAg}$, $\times 200$ ). (d) The mHBcAg type showed 50\% HBcAg expression in the nucleus and $50 \% \mathrm{HBcAg}$ expression in the cytoplasm of the hepatocytes (immunohistochemical stain for $\mathrm{HBcAg}, \times 200$ ).

response (VR) was defined as a serum HBV DNA level of less than $500 \mathrm{IU} / \mathrm{mL}$. Biochemical response (BR) was defined as ALT and AST levels returning to normal. The following cases were discontinued during treatment: patients who had difficulty tolerating the side effects and patients who refused to continue antiviral therapy. The following cases were discontinued during 24 weeks follow-up: patients who had an elevation of ALT levels $>10$ times ULN; patients who refused to continue following up and switch to nucleoside analogues (NAs) therapy.

2.4. Statistical Analysis. All statistical analyses were performed using SPSS 22.0 software (IBM Co., Armonk, NY, USA). Continuous variables are presented as the mean \pm standard deviation (or median and range), and the MannWhitney $U$ test was used to compare nonparametric 


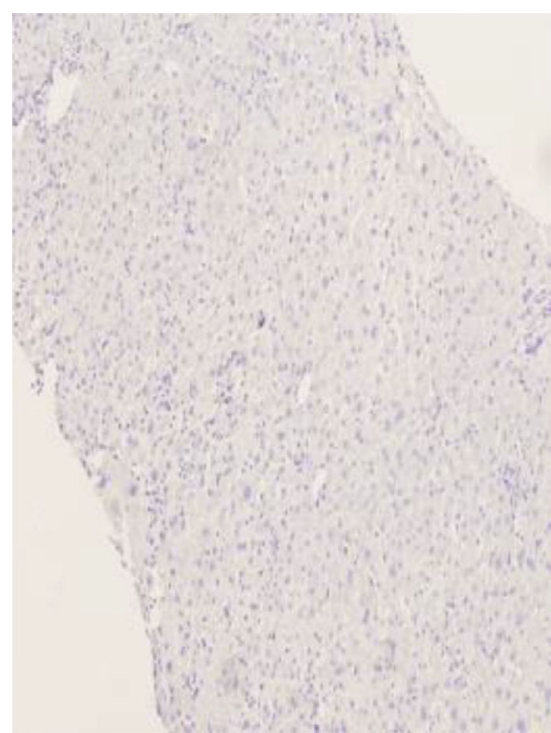

(a)

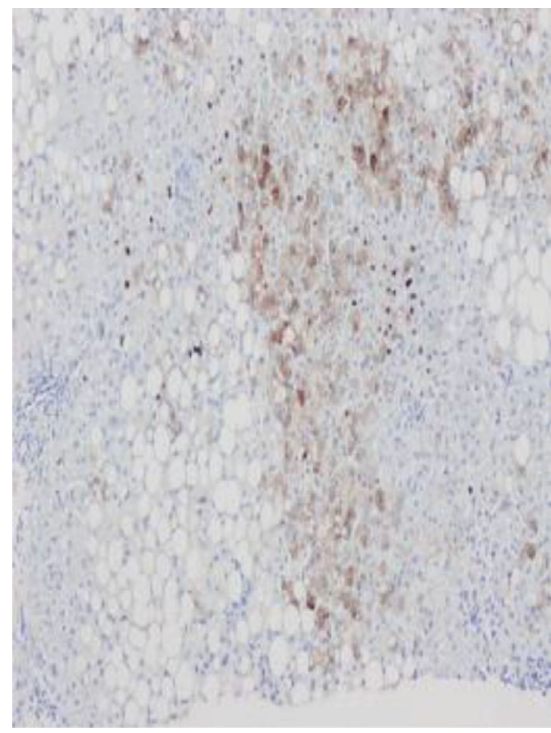

(c)

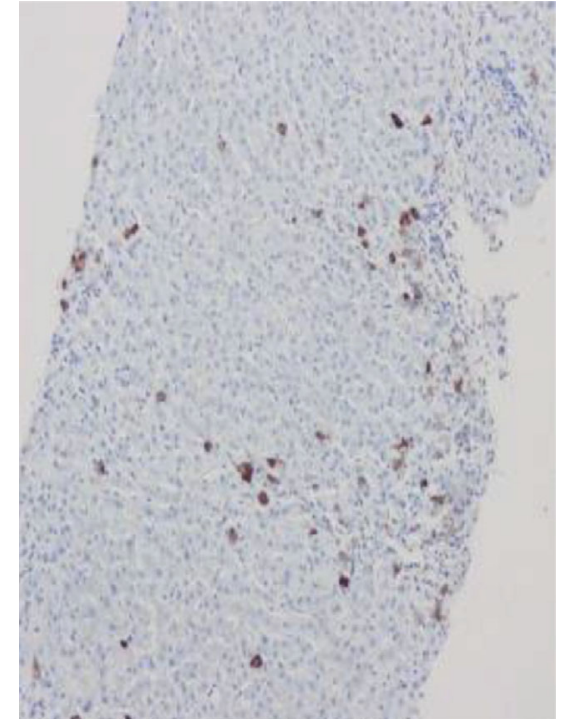

(b)

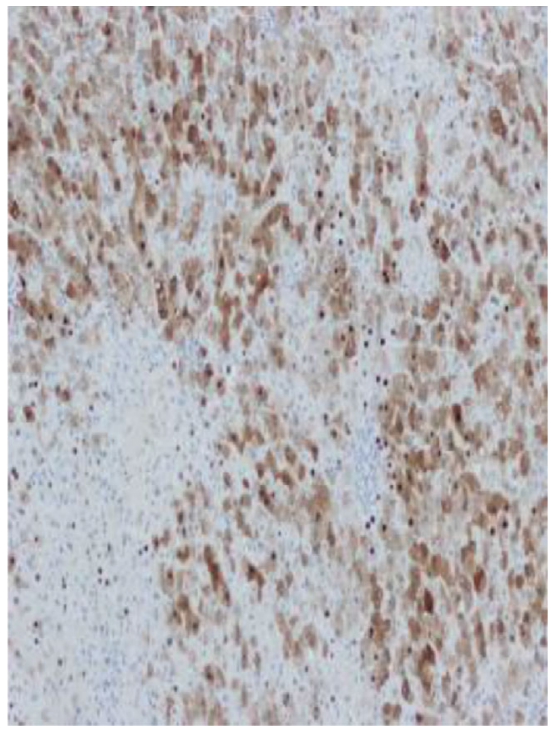

(d)

FIGURE 3: HBcAg expression intensity was classified into four types in this study. (a) The 0 point group showed HBcAg expression in less than $5 \%$ of hepatocytes (immunohistochemical stain for $\mathrm{HBcAg}, \times 200$ ). (b) The 1 point group showed $\mathrm{HBcAg}$ expression in $5 \%-33 \%$ of hepatocytes (immunohistochemical stain for HBcAg, $\times 200$ ). (c) The 2 point group showed HBcAg expression in $34 \%-66 \%$ of hepatocytes (immunohistochemical stain for $\mathrm{HBcAg}$, $\times 200$ ). (d) The 3 point group showed $\mathrm{HBcAg}$ expression in over 66\% of hepatocytes (immunohistochemical stain for $\mathrm{HBcAg}, \times 200$ ).

continuous variables. Categorical variables are expressed as the frequency and percentage and were analysed with the chi-square test. The correlation between groups and the results was analysed by Spearman's rank correlation analysis. Multivariate logistic regression was used to determine the independent predictors of the relationship between the complete response rate and related factors. A two-sided $P$ value $<0.05$ was considered statistically significant.

\section{Results}

3.1. Baseline Characteristics of Different Degrees of Hepatic Steatosis in CHB Patients. The baseline characteristics are listed in Table 1. The patients were divided into four groups according to their degrees of hepatic steatosis. CHB patients with hepatic steatosis were more likely to be male, be aged $>40$ years, have a BMI $>23 \mathrm{~kg} / \mathrm{m}^{2}$, have hyperuricaemia, have higher levels of TC, TG, GLU, and UA, and have lower levels of ALT and AST, as shown in Table 1.

Continuous variables are expressed as the mean $\pm \mathrm{SD}$ or median (interquartile range), and categorical variables are described by counts and proportions. Pearson's chi-square test and the Student's $t$-test were used for statistical analysis. Abbreviations: TC: total cholesterol; TG: triglycerides; UA: uric acid; GLU: glucose; ALT: alanine aminotransferase; AST: aspartate aminotransferase, HBV: hepatitis B virus; BMI: body mass index. 


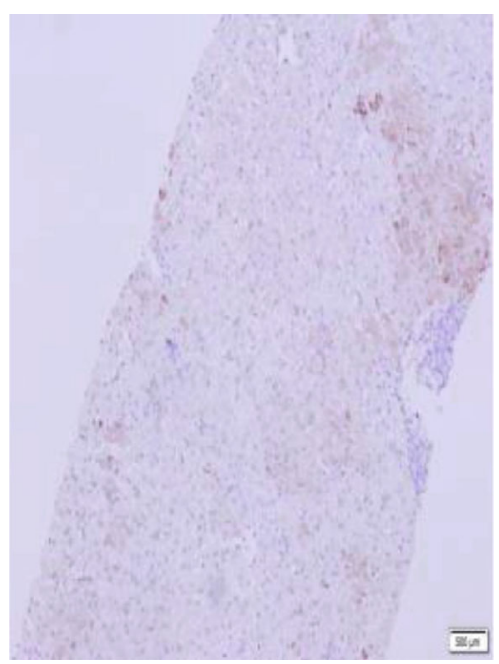

(a)

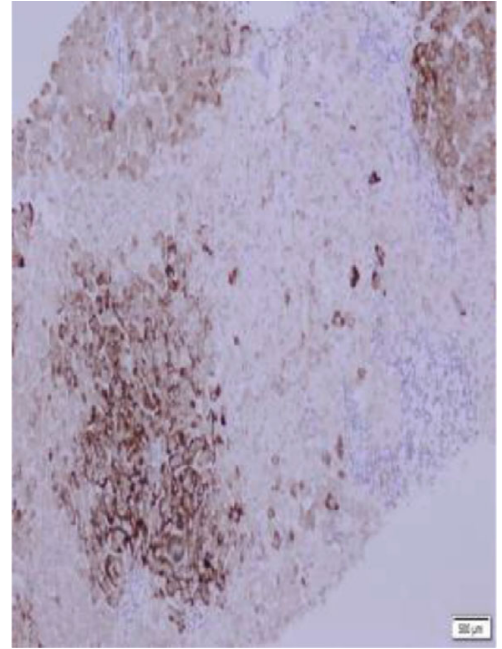

(b)

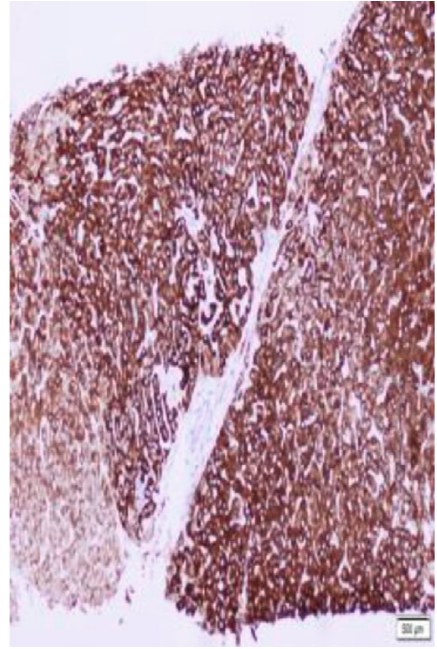

(c)

Figure 4: HBsAg expression intensity was classified into three types in this study. (a) The 1 point group showed HBsAg expression in 5\%-33\% of hepatocytes (immunohistochemical stain for HBsAg, $\times 200$ ). (b) The 2 point group showed HBsAg expression in 34\%-66\% of hepatocytes (immunohistochemical stain for HBsAg, $\times 200$ ). (c) The 3 point group showed HBsAg expression in over $66 \%$ of hepatocytes (immunohistochemical stain for HBsAg, ×200).

3.2. Correlation between Hepatic Steatosis and Hepatic Histological Necroinflammation, Fibrosis Stage. The baseline hepatic histological necroinflammation and hepatic steatosis stages were negatively correlated. The more severe the hepatic steatosis was, the less severe the liver inflammation activity (Table 2). There was no correlation between the degree of liver fibrosis and hepatic steatosis (Table 3 ).

The categorical variables are described by counts and proportions. The Pearson chi-square test, Spearman's rank correlation analysis, and coefficient of contingency were used for the statistical analysis.

The categorical variables are described by counts and proportions. The Pearson chi-square test, Spearman's rank correlation analysis, and coefficient of contingency were used for the statistical analysis.

\subsection{Risk Factors Associated with Significant Histological} Abnormalities. Clinical parameters (sex, age, disease course, baseline TC, baseline TG, metabolic syndrome, baseline ALT, baseline AST, baseline HBV DNA, BMI $>23 \mathrm{~kg} / \mathrm{m}^{2}$, baseline UA, and GLU) were analysed by multivariate regression and were correlated with liver histological abnormalities (Table 4$)$. BMI $>23 \mathrm{~kg} / \mathrm{m}^{2} \quad(P<0.001)$ was independently associated with the presence of hepatic steatosis in $\mathrm{CHB}$ patients. Baseline AST $(P=0.029)$ and baseline UA $(P=0.040)$ were independently associated with significant hepatic steatosis in CHB patients. Baseline AST $(P=0.038)$ was independently associated with significant fibrosis in CHB patients.

\subsection{Correlation between Hepatic Steatosis and the Rates of} $V R, B R$, and CR after PEG-IFN $\alpha-2 a$ Treatment. The VR, $\mathrm{BR}$, and $\mathrm{CR}$ rates were observed in the four groups after 48 weeks of treatment. The rates of VR, BR, and CR differed significantly between the four groups $(P<0.05)$. The more severe the hepatic steatosis was, the lower the VR, BR, and CR rates (Table 5).

Categorical variables are described by counts and proportions. The Pearson chi-square test was used for the statistical analysis.

3.5. Correlation between Hepatic Steatosis and the Rates of $V R, B R$, and CR after 24 Weeks of Follow-Up. During 24 weeks follow-up, there were thirteen patients who had an elevation of ALT levels $>10$ times ULN discontinued follow-up and switch to NAs therapy and seven patients intended to NA therapy so drop out continue the follow-up. 206 patients finished the follow-up.

The VR, BR, and $\mathrm{CR}$ rates were observed in the four groups after 24 weeks of follow-up. The rates of VR, BR, and $\mathrm{CR}$ differed significantly between the four groups $(P<0.05)$. The more severe the hepatic steatosis was, the lower the VR, BR, and CR rates (Table 6).

Categorical variables are described by counts and proportions. The Pearson chi-square test was used for the statistical analysis.

3.6. Correlation between Hepatic Steatosis and HBcAg and $H B s A g$ Expression. The four degrees of hepatic steatosis have different $\mathrm{HBcAg}$ expression patterns. The rate of $\mathrm{HBcAg-}$ negative and c-HBcAg was $43.70 \%$ in the S0 group, whereas it was $31.58 \%, 31.82 \%$, and $33.33 \%$ in the S1, S2, and S3 groups, respectively $(P=0.015)$ (Table 7$)$. However, the expression levels of $\mathrm{HBsAg}$ and $\mathrm{HBcAg}$ did not differ significantly between groups (Tables 8 and 9 ).

\section{Discussion}

In this study, we compared the baseline clinical data of $\mathrm{CHB}$ patients with different degrees of hepatic steatosis. $\mathrm{CHB}$ patients with hepatic steatosis were more likely to 
TABLE 1: Baseline characteristics of 226 chronic hepatitis B patients.

\begin{tabular}{|c|c|c|c|c|c|c|}
\hline \multirow{2}{*}{ Variable } & \multicolumn{4}{|c|}{ Degrees of hepatic steatosis } & \multirow{2}{*}{$F$ value } & \multirow{2}{*}{$P$ value } \\
\hline & S0 $(N=119)$ & $\mathrm{S} 1(N=76)$ & $\mathrm{S} 2(N=22)$ & $\mathrm{S} 3(N=9)$ & & \\
\hline Male $(n(\%))$ & $82(68.91 \%)$ & $65(85.53 \%)$ & $21(95.46 \%)$ & $9(100 \%)$ & 14.828 & 0.002 \\
\hline Age & $29.50 \pm 5.47$ & $31.46 \pm 8.40$ & $29.41 \pm 6.19$ & $27.00 \pm 7.38$ & 2.063 & 0.106 \\
\hline Age $>40(n(\%))$ & $6(5.04 \%)$ & $15(19.74 \%)$ & $1(4.55 \%)$ & $0(0 \%)$ & 13.28 & 0.004 \\
\hline Course of disease & $8.57 \pm 5.96$ & $8.17 \pm 5.29$ & $8.98 \pm 5.84$ & $10.0 \pm 9.50$ & 0.327 & 0.806 \\
\hline $\mathrm{TC}$ & $4.68 \pm 0.80$ & $5.15 \pm 0.94$ & $5.05 \pm 1.11$ & $5.43 \pm 1.25$ & 5.703 & 0.001 \\
\hline TG & $0.56 \pm 0.45$ & $1.34 \pm 0.68$ & $1.42 \pm 0.44$ & $1.76 \pm 0.63$ & 13.180 & 0.001 \\
\hline UA & $345.78 \pm 69.38$ & $370.10 \pm 78.07$ & $381.22 \pm 101.58$ & $429.69 \pm 65.93$ & 4.271 & 0.006 \\
\hline Hyperuricaemia $(n(\%))$ & $18(15.13 \%)$ & $17(22.37 \%)$ & $9(40.91 \%)$ & $6(66.67 \%)$ & 18.255 & 0.001 \\
\hline Metabolic syndrome $(n(\%))$ & $1(0.84 \%)$ & $3(3.95 \%)$ & $1(4.55 \%)$ & $1(11.11 \%)$ & 4.537 & 2.063 \\
\hline GLU & $4.98 \pm 0.50$ & $5.35 \pm 0.63$ & $5.21 \pm 0.36$ & $5.67 \pm 1.05$ & 9.404 & 0.001 \\
\hline ALT & $257.39 \pm 175.17$ & $186.14 \pm 162.63$ & $182.45 \pm 108$ & $137.44 \pm 61.88$ & 4.347 & 0.005 \\
\hline AST & $126.72 \pm 79.55$ & $88.07 \pm 67.82$ & $76.95 \pm 39.30$ & $57.44 \pm 24.10$ & 7.560 & 0.001 \\
\hline HBVlog10 (IU/mL) DNA & $7.17 \pm 0.82$ & $6.96 \pm 0.98$ & $7.23 \pm 1.15$ & $7.47 \pm 0.69$ & 1.224 & 0.302 \\
\hline HBsAg $(\log I U / m L)$ & $4.12 \pm 1.14$ & $4.04 \pm 0.91$ & $4.23 \pm 1.18$ & $4.19 \pm 1.13$ & 1.273 & 0.283 \\
\hline HBV genotype & & & & & 1.00 & 0.616 \\
\hline B & 35 (29.41\%) & $23(30.26 \%)$ & $7(31.83 \%)$ & $3(33.33 \%)$ & & \\
\hline $\mathrm{C}$ & $78(65.55 \%)$ & $50(65.79 \%)$ & $14(63.64 \%)$ & $6(66.67 \%)$ & & \\
\hline Mix & $6(5.04 \%)$ & $3(3.95 \%)$ & $1(4.55 \%)$ & 0 & & \\
\hline BMI & $20.87 \pm 1.96$ & $24.98 \pm 3.82$ & $24.91 \pm 3.91$ & $25.78 \pm 3.44$ & 36.996 & 0.001 \\
\hline $\mathrm{BMI}>23 \mathrm{~kg} / \mathrm{m}^{2}$ & $14(11.76 \%)$ & $54(71.05 \%)$ & $14(63.64 \%)$ & $7(77.77 \%)$ & 80.932 & 0.001 \\
\hline
\end{tabular}

TABLE 2: The correlation between baseline hepatic histological necroinflammation and baseline hepatic steatosis in each group.

\begin{tabular}{lcccccc}
\hline Feature & $\begin{array}{c}\text { Total } \\
\text { cases }\end{array}$ & G1 & G2 & $\geq \mathrm{G} 3$ & $\begin{array}{c}\text { Spearman's rank } \\
\text { correlation } \\
\text { analysis }\end{array}$ & $\begin{array}{c}\text { Coefficient of } \\
\text { contingency }\end{array}$ \\
\hline S0 & 119 & 2 & 74 & 43 & & \\
S1 & 76 & 1 & 55 & 20 & 0.036 & -0.140 \\
S2 & 22 & 0 & 16 & 6 & & \\
S3 & 9 & 0 & 9 & 0 & & \\
\hline
\end{tabular}

TABLE 3: The correlation between baseline hepatic fibrosis stage and baseline hepatic steatosis degree.

\begin{tabular}{lcccccc}
\hline Feature & $\begin{array}{c}\text { Total } \\
\text { cases }\end{array}$ & F1 & F2 & $\geq$ F3 & $\begin{array}{c}\text { Spearman's rank } \\
\text { correlation analysis }\end{array}$ & $\begin{array}{c}\text { Coefficient of } \\
\text { contingency }\end{array}$ \\
\hline S0 & 119 & 69 & 40 & 10 & & \\
S1 & 76 & 50 & 20 & 6 & 0.202 & -0.085 \\
S2 & 22 & 2 & 6 & 14 & & \\
S3 & 9 & 7 & 2 & 0 & & \\
\hline
\end{tabular}

have higher levels of TC, TG, GLU, UA, and BMI; be of themale sex; be aged $>40$ years; have hyperuricaemia and a $\mathrm{BMI}>23 \mathrm{~kg} / \mathrm{m}^{2}$; and have significantly lower ALT and AST levels than those without hepatic steatosis. The UA and TG levels and the incidence of hyperuricaemia gradually increased with the degree of hepatic steatosis, whereas the proportion of patients over 40 years old gradually decreased. The combined presence of $\mathrm{CHB}$ and hepatic steatosis was associated with metabolic disorders and had caused diabetes mellitus, atherosclerosis, and cardiovascular and cerebrovascular events [16-18]. The proportion of patients aged $>40$ years gradually decreased with higher degrees of hepatic steatosis, suggesting that lipocytes were necrotic and hepatic fibrous tissue gradually proliferated with age.

The multivariate logistic regression analysis showed that a $\mathrm{BMI}>23 \mathrm{~kg} / \mathrm{m}^{2}$ was an independent risk factor for $\mathrm{CHB}$ with hepatic steatosis, which suggests that overweight or obesity is an important feature of $\mathrm{CHB}$ patients with hepatic steatosis and has caused or may be related to increased insulin resistance $[19,20]$. Insulin resistance can impair the ability of surrounding tissues to absorb and utilize glucose, resulting in the deposition of a large amount of TG in the liver, which leads to the formation of hepatic steatosis. Furthermore, the multivariate logistic regression analysis showed that baseline levels of AST and UA were independent risk factors for $\mathrm{CHB}$ with significant hepatic steatosis (hepatic steatosis $\geq$ S2), and baseline AST levels were independent risk factors for significant liver fibrosis (fibrosis $\geq$ F2). There was no significant difference in HBV DNA titres between the four groups, which indicates that HBV replication was not associated with the presence of hepatic steatosis.

In this study, we present our findings that a higher degree of hepatic steatosis is associated with a lower level of inflammation in the liver tissue; moreover, there was no correlation 
TABLE 4: Multivariate analysis of clinical parameters independently associated with significant histological abnormalities.

\begin{tabular}{lccc}
\hline Patient characteristics & Clinical parameters & OR (95\% CI) & $P$ value \\
\hline Presence of hepatic steatosis & BMI $>23 \mathrm{~kg} / \mathrm{m}^{2}$ & $5.481(2.992-10.041)$ & 0.001 \\
& Baseline AST & $0.978(0.959-0.998)$ & 0.029 \\
Presence of significant hepatic steatosis $(\geq \mathrm{S} 2)$ & Baseline UA & $1.006(1.000-1.012)$ & 0.040 \\
Presence of significant fibrosis $(\geq \mathrm{F} 2)$ & Baseline AST & $1.006(1.000-1.012)$ & 0.038 \\
\hline
\end{tabular}

TABLE 5: The virological response, biochemical response, and complete response in the four groups after 48 weeks of treatment.

\begin{tabular}{lcccc}
\hline Groups & Cases & The rate of VR & The rate of BR & The rate of CR \\
\hline CHB with S0 & 119 & $42.9 \%$ & $75.6 \%$ & $28.6 \%$ \\
CHB with S1 & 76 & $30.3 \%$ & $40.9 \%$ & $14.5 \%$ \\
CHB with S2 & 22 & $18.2 \%$ & $31.8 \%$ & $9.1 \%$ \\
CHB with S3 & 9 & $0 \%$ & $22.2 \%$ & $0 \%$ \\
$F$ value & & 11.777 & 32.228 & 9.552 \\
$P$ value & & 0.007 & 0.001 & 0.018 \\
\hline
\end{tabular}

TABLE 6: The virological response, biochemical response, and complete response in the four groups after 24 weeks of follow-up.

\begin{tabular}{lcccc}
\hline Groups & Cases & The rate of VR & The rate of BR & The rate of CR \\
\hline CHB with S0 & 110 & $40.0 \%$ & $68.2 \%$ & $34.5 \%$ \\
CHB with S1 & 72 & $26.3 \%$ & $27.1 \%$ & $20.8 \%$ \\
CHB with S2 & 18 & $16.7 \%$ & $16.7 \%$ & $11.1 \%$ \\
CHB with S3 & 6 & $0 \%$ & 25.988 & $0 \%$ \\
$F$ value & & 8.505 & 0.001 & 8.390 \\
$P$ value & & 0.029 & & 0.032 \\
\hline
\end{tabular}

TABLE 7: The difference in HBcAg expression patterns in different degrees of steatosis.

\begin{tabular}{|c|c|c|c|c|c|c|}
\hline Degrees of steatosis & Total cases & HBcAg-negative group & c-HBcAg group & cd-HBcAg group & m-HBcAg group & Pearson's chi-square test \\
\hline So & 119 & 5 & 47 & 63 & 4 & \multirow{4}{*}{0.015} \\
\hline S1 & 76 & 10 & 14 & 48 & 4 & \\
\hline S2 & 22 & 3 & 4 & 13 & 2 & \\
\hline S3 & 9 & 0 & 3 & 5 & 1 & \\
\hline
\end{tabular}

TABLE 8: The difference in HBsAg expression levels in different degrees of steatosis.

\begin{tabular}{lccccc}
\hline Degrees of steatosis & Total cases & 1 & 2 & 3 & Rank test \\
\hline S0 & 119 & 8 & 10 & 101 & \\
S1 & 76 & 3 & 13 & 60 & \\
S2 & 22 & 1 & 5 & 16 & 0.085 \\
S3 & 9 & 0 & 1 & 8 & \\
\hline
\end{tabular}

between the degree of liver fibrosis and the degree of hepatic steatosis. One domestic study [15] showed that the mechanism by which a higher degree of hepatic steatosis results in lower liver inflammation may be the ability of hepatic steatosis to weaken the expression intensity of HBsAg and HBcAg in liver tissue. Thus, the ability of viral proteins to activate cellular immune responses is reduced, which reduces the destruction of infected hepatocytes and causes slight liver inflammation.
TABle 9: The difference in HBcAg expression levels in different degrees of steatosis.

\begin{tabular}{lcccccc}
\hline Degrees of steatosis & Total cases & 0 & 1 & 2 & 3 & Rank test \\
\hline S0 & 119 & 8 & 28 & 20 & 63 & \\
S1 & 76 & 10 & 13 & 17 & 36 & \\
S2 & 22 & 3 & 4 & 5 & 10 & 0.257 \\
S3 & 9 & 0 & 1 & 3 & 5 & \\
\hline
\end{tabular}

However, our study investigated the difference in expression levels of $\mathrm{HBsAg}$ and $\mathrm{HBcAg}$ in different groups and found that there was no significant difference in the expression levels of $\mathrm{HBsAg}$ and $\mathrm{HBcAg}$ between groups with different severities of hepatic steatosis. Furthermore, we demonstrated that $\mathrm{HBcAg}$ expression patterns were related to hepatic steatosis. In the group without steatosis, $43.70 \%$ of patients were $\mathrm{HBcAg}$-negative and $\mathrm{c}-\mathrm{HBcAg}$, whereas 
$31.77 \%$ of patients with steatosis were $\mathrm{HBcAg}$-negative and c-HBcAg. One study [14] showed that the expression patterns of $\mathrm{HBcAg}$-negative and $\mathrm{c}-\mathrm{HBcAg}$ in the liver tissues of $\mathrm{CHB}$ patients usually occurred in the stage of immune clearance and active liver inflammation, whereas the cd$\mathrm{HBcAg}$ group and the m-HBcAg group were often in the stage of immune tolerance and had mild liver inflammation, resulting in a lower antiviral effect. Therefore, the effect of PEG-IFN $\alpha$-2a in CHB patients with hepatic steatosis was poor. Our study compared the rates of VR, BR, and CR among the four degrees of hepatic steatosis after 48 weeks of treatment and 24 weeks of follow-up. There was a significant difference between the four groups. With the increase in the degree of steatosis, the rates of VR, BR, and CR decreased significantly. Therefore, we should take effective measures for the prevention and treatment of hepatic steatosis to improve the efficacy of PEG-IFN $\alpha$-2a.

In conclusion, ALT and AST levels and the inflammatory activity of liver tissue in $\mathrm{CHB}$ patients with steatosis were significantly lower than those in patients without steatosis; this result may be due to the low expression of the HBcAgnegative and $\mathrm{c}-\mathrm{HBcAg}$ type in liver tissue that leads to a poor immune response, eventually causing a poor antiviral effect. Antiviral therapy with PEG-IFN $\alpha-2 \mathrm{a}$ is not appropriate for $\mathrm{CHB}$ patients with hepatic steatosis. Conversely, hepatic steatosis is mainly related to sex, baseline TC, TG, UA, GLU, and BMI but is unrelated to HBV DNA load. Therefore, we can improve the clinical efficacy of PEG-IFN $\alpha$ - $2 \mathrm{a}$ in CHB patients with hepatic steatosis by weight control, alcohol abstinence, diet adjustment, and insulin resistance improvement.

There are several limitations of the present study. We evaluated the efficacy of PEG-IFN $\alpha$-2a treatment for 48 weeks and 24 weeks of drug withdrawal without evaluating the sustained efficacy of 48 weeks of drug withdrawal because several patients needed antiviral therapy again due to the significantly elevated serum ALT and AST levels after the drug withdrawal for more than 24 weeks, which would have led to a large dropout rate. Our study was performed in a single centre and lacked sufficient samples, particularly in the severe steatosis group. Therefore, a multicentre, a balanced number of each group, and a large-sample study should be conducted in the future.

\section{Data Availability}

The data used to support the findings of this study are included within the article.

\section{Conflicts of Interest}

The authors declare that they have no conflicts of interest.

\section{Acknowledgments}

This study was supported by the National Natural Science Foundation of China (No. 81673660, No. 81873242), the National Science and technology major special project (No: 2017zx10202201), the Fujian Province Health and Family Planning Medical Innovation Research Talent Training Pro- ject (No. 2018-CXB-28; No. 2018-2-68), the Xiamen Science and Technology Program Grant (No. 3502Z20174028), and the joint research of Xiamen Important and Major Disease Project (No. 3502Z20179047), the sixth group of experts in traditional Chinese medicine in China (2017).

\section{References}

[1] R. Pais, E. Rusu, and V. Ratziu, "The Impact of Obesity and Metabolic Syndrome on Chronic Hepatitis B and DrugInduced Liver Disease," Clinics in Liver Disease, vol. 18, no. 1, pp. 165-178, 2014.

[2] G. C. De Andrade, L. H. Fujise, J. E. De Santana, and F. Oliveira, "Non-alcoholic fatty liver disease (NAFLD) in different populations: a clinical and epidemiological study - sample of São José do Rio Preto," Revista da Associação Médica Brasileira, vol. 62, no. 3, pp. 218-226, 2016.

[3] J. P. Shi, J. G. Fan, R. Wu, X. Q. Gao, L. Zhang, and H. Wang, "Incidence and risk factors of hepatocyte steatosis in patients with chronic hepatitis B," Chinese journal of hepatopathy, vol. 16, no. 7, pp. 519-523, 2008.

[4] A. Marengo, R. I. K. Jouness, and E. Bugianesi, "Progression and natural history of nonalcoholic fatty liver disease in adults," Clinics in Liver Disease, vol. 20, no. 2, pp. 313-324, 2016.

[5] E. Altlparmak, S. Koklu, M. Yalinkilic et al., "Viral and host causes of fatty liver in chronic hepatitis B," World Journal of Gastroenterology, vol. 11, no. 20, pp. 3056-3059, 2005.

[6] L. Y. Chen and J. P. Shi, "The relationship between chronic hepatitis b and metabolic syndrome," Chinese journal of hepatopathy, vol. 17, no. 11, pp. 807-808, 2009.

[7] Y.-L. Cheng, Y.-J. Wang, W.-Y. Kao et al., "Inverse association between hepatitis B virus infection and fatty liver disease: a large-scale study in populations seeking for check-up," PLoS One, vol. 8, no. 8, pp. 519-523, 2008.

[8] L. Xu, P. Li, Q. Shi, and Y. Mi, "Impact of liver steatosis on the curative effect of pegylated interferon- $\alpha-2 \mathrm{a}$ in patients with chronic hepatitis B," Chinese journal of hepatopathy, vol. 23, no. 2, pp. 99-102, 2015.

[9] C. H. Xiao, X. H. Li, C. L. Zhang, and X. Y. Jian, "Efficacy of adefovir dipivoxil in treatment of chronic hepatitis B patients with non-alcoholic fatty liver disease," Journal clinical hepatopathy, vol. 31, no. 2, pp. 266-268, 2015.

[10] X. Jin, Y. P. Chen, Y. M. Yi-da Yang, L. Zheng, and C. Q. Xu, "Association between hepatic steatosis and entecavir treatment failure in Chinese patients with chronic hepatitis B," PLoS One, vol. 7, no. 3, pp. 198-201, 2012.

[11] H. J. Dong, Y. Q. Mi, J. Wang et al., "Influence of chronic hepatitis B complicated with hepatic steatosic to response of telbivudine therapy," Chinese journal of New Drugs and Clinic, vol. 12, no. 10, pp. 759-761, 2008.

[12] P. J. Scheuer, "Classification of chronic viral hepatitis: a need for reassessment," Journal of Hepatology, vol. 13, p. 372e4, 1991.

[13] L. Fatty, "Alcoholic liver disease study group of the Chinese Liver Disease Association. Nonalcoholic fatty liver disease diagnosis and treatment guidelines," Chinese journal of hepatopathy, vol. 18, pp. 163-166, 2010.

[14] H. Liang, J. Tang, J. Yang et al., "Hepatitis B core antigen expression in hepatocytes reflects viral response to PegIFN $\alpha-2 \mathrm{a}$ in HBeAg-positive chronic hepatitis B patients," 
International Journal of Clinical and Experimental Pathology, vol. 9, no. 3, pp. 3563-3570, 2016.

[15] M. I. Yuqiang, Y. Liu, X. U. Liang et al., "Relationship between hepatic steatosis and $\mathrm{HBsAg}, \mathrm{HBcAg}$ in patients with chronic hepatitis B," Chinese Journal of digestion, vol. 32, no. 5, pp. 316-319, 2012.

[16] Z. Karacaer, "Non-alcoholic fatty liver disease in chronic hepatitis B patients," The Turkish Journal of Gastroenterology, vol. 27, no. 4, pp. 395-396, 2016.

[17] K. G. Alberti, R. H. Eckel, S. M. Grundy et al., "Harmonizing the metabolic syndrome: a joint interim statement of the international diabetes federation task force on epidemiology and prevention; National Heart, Lung, and Blood Institute; American Heart Association; World Heart Federation; International Atherosclerosis Society; and International Association for the Study of Obesity," Circulation, vol. 120, no. 16, pp. 16401645, 2009.

[18] R. Kwok, K. C. Choi, G. L. H. Wong et al., "Screening diabetic patients for non-alcoholic fatty liver disease with controlled attenuation parameter and liver stiffness measurements: a prospective cohort study," Gut, vol. 65, no. 8, pp. 1359-1368, 2016.

[19] J. G. Fan, S. U. Kim, and V. W. Wong, "New trends on obesity and NAFLD in Asia," Journal of hepatology, vol. 67, no. 4, pp. 862-873, 2017.

[20] E. Koroglu, B. Canbakan, K. Atay et al., "Role of oxidative stress and insulin resistance in disease severity of nonalcoholic fatty liver disease," The Turkish Journal of Gastroenterology, vol. 27, no. 4, pp. 361-366, 2016. 